\title{
Case - Penile verrucous epithelial hyperplasia
}

\author{
Jonathan Fadel $^{1}$; Michele Lodde ${ }^{1}$; Louis-Phillipe Gagnon ${ }^{2}$; Sylvie Fraitag ${ }^{3}$; Katherine \\ Moore $^{1}$ \\ ${ }^{1}$ Division of Urology, Department of Surgery, CHU de Québec-Université Laval, Quebec City, QC, \\ Canada; ${ }^{2}$ Department of Molecular Biology, Clinical Biochemistry and Pathology, CHU de Québec- \\ Université Laval, Quebec City, QC, Canada; ${ }^{3}$ Department of Anatomy and Pathology, Necker-Enfants \\ Malades Hospital, Paris, France
}

Cite as: Fadel J, Lodde M, Gagnon L-P, et al. Case - Penile verrucous epithelial hyperplasia. Can Urol Assoc J 2021 May 11; Epub ahead of print. http://dx.doi.org/10.5489/cuaj.7027

Published online May 11, 2021

Correspondence: Dr. Katherine Moore, Division of Urology, CHU de QuébecUniversité Laval, Quebec City, QC, Canada; katherine.moore.1@ulaval.ca

\section{$* * *$ \\ Introduction}

Masses of the penis are uncommon, especially in the pediatric population, and they need to be promptly assessed and biopsied. We present what we believe to be the first case of rapidly recurring case of glans and foreskin verrucous epithelial hyperplasia, a premalignant lesion. The young patient, a 13-year-old uncircumcised teenager, presented an itching lesion growing over four months and rapidly recurring after resection.

\section{Case report}

A 13-year-old uncircumcised teenager, with a past medical history of asthma, autism, and attention deficit hyperactivity disorder, presented to our department for the evaluation of a penile lesion after initial evaluation by dermatology. The mass was first noticed on the glans 18 months prior to the consultation but was disregarded by the patient. However, he finally requested medical attention because the lesion rapidly progressed over a fourmonths period. Clinically, the chief complaint was pruritus with neither pain, discharge nor urinary symptoms. Initial physical exam showed a $1.5 \mathrm{~cm}$ beige ulcerative and crateriform lesion on the right lateral side of the glans (Fig.1). Testes were normal in size and shape as well as retraction of the foreskin for this Tanner 5 teenager. There was no palpable inguinal lymph node. Considering the absence of other risk factors such as infection or phimosis, dermatologist considered the lesion to be the results of chronic 
scratching. Topical antibiotic treatment was attempted but was not effective. Biopsy without sedation was not feasible. Therefore, we performed a first surgical excision of the $1.5 \times 1.0 \times 0.5 \mathrm{~cm}$ glans tumor and of a $0.5 \times 0.5 \times 0.3 \mathrm{~cm}$ satellite tumor on the inner foreskin. The main lesion was sent for frozen section, which showed a benign verrucous and papillomatous epithelial lesion and negative margins. The final local and revised histology reports (by pathologist at Necker-Enfants Malades Hospital, Paris) described a marked epidermal hyperplasia with numerous dyskeratotic cells scattered at different levels of this epidermis. There was no atypia nor mitotic figures or koilocytes (Fig. 2). Human papillomavirus (HPV) immunohistochemistry typing and mycosis cultures were negative.

At one-month follow-up, we noted two new lesions near the previous surgical excision area (Fig. 3). Masses were removed with deeper and larger surgical margins. Macroscopic examination of the specimen showed beige masses of $0.4 \times 0.3 \times 0.2 \mathrm{~cm}$ and $1.8 \times 1.3 \times 0.6 \mathrm{~cm}$. Histologic slides showed similar findings and the Necker-Enfants Malades Hospital's pathologist concluded for a diagnosis of "penile verrucous epithelial hyperplasia."

Two months later, a new lesion appeared on the right side of the glans at great distance from the previous resection sites. Partial penectomy with glans resection and entire corpora cavernosa preservation was performed (Fig. 4). Surgical margins were clear. Histopathology showed similar findings to what was previously seen.

After 18 months of follow-up, patient is well without any urinary problems. Penile sensitivity is intact without residual pain nor erectile dysfunction. No shaft relapse has been seen.

\section{Discussion}

We present a patient with a very atypical clinical presentation for a penile lesion., considering his young age and the absence of risk factors. He was not sexually active, non-smoker, uncircumcised without phimosis and HPV tested negative. Furthermore, the lesion relapsed multiples times in a short period following surgeries, which makes this case even more unusual.

Pediatric penile lesions differential diagnosis includes infectious and sexually transmitted diseases, inflammatory conditions, traumatic causes, pre-malignant and malignant lesions, even though they remain very rare in children. Cancers of the penis and pre-malignant penile lesions are considered disease affecting older patients, mostly men aged between 50 and 70 years old. ${ }^{1}$ The incidence in Europe and in the United States is approximately $1 / 10000^{2}$ with incidence increasing with age and with the prevalence of HPV. Squamous cell carcinoma is the most common pathology type. Risk factors are numerous and include untreated phimosis that induces chronic inflammation, ${ }^{3} \mathrm{HPV}$ 
infection and penile trauma, phototherapy, psoriasis, smoking, poor hygiene, low social and economic status, and marital status. ${ }^{4}$

Based on the current literature, penile cancer has been rarely documented in the pediatric population. Penile rhabdomyosarcoma (RMS) in a 3-year-old child ${ }^{5}$ and 16year-old boy ${ }^{6}$ have been reported, with a painless mass that can be treated by surgery, chemotherapy, and radiation therapy. Congenital penile teratoma ${ }^{7}$ and glomus tumor of the penis ${ }^{8}$ in pediatric patients have been registered. In our report, the final diagnosis was verrucous epithelial hyperplasia (VEH) that is usually a premalignant lesion ${ }^{9}$ that can transform into verrucous carcinoma (VC), a very well differentiated variant of squamous cell carcinoma (SCC). It has been published as an oral mucosal lesion but has never been reported in genital tumors neither in the pediatric population. ${ }^{10}$ This type of lesion has been associated to tobacco and HPV.

\section{Conclusions}

Although this is a unique case, the first reported in a teenager and in the genital apparatus, verrucous epithelial hyperplasia should be considered in the differential diagnosis of a penile lesion.

We strongly believe that any penile mass should be rapidly investigated, particularly in children without risk factors, by specialized dermatologist and urologist to provide a prompt and appropriate treatment. 


\section{References}

1. Douglawi A, Masterson T.A. Updates on the epidemiology and risk factors for penile cancer. Translational andrology and urology 2017; 6(5):785-90.

2. Ries LA, Hankey BF, Edwards BK. SEER cancer statistics review. National Cancer Institute, National Institutes of Health Publication 1973-1996;90-2789.

3. Larke N.L, Thomas S.L., dos Santos Silva I et al. Male circumcision and penile cancer: a systematic review and meta-analysis. Cancer causes \& control 2011;22(8), 1097-110.

4. Morrison, B.F, Risk Factors and Prevalence of Penile Cancer. The West Indian Medical Journal 2014;63(6), 559-60.

5. Palacios-Acosta JM, Venzor-Pérez IG, Hernández-Arrazola D et al. Rhabdomyosarcoma of the penis in a boy. Report of a case. Acta Pediatr Mex. 2013;34(1):11-15.

6. Venkov G, Khristova S, Mavrov, Kh. Rhabdomyosarcoma of the penis. Khirurgiia 2006;(6), 59-61.

7. Hu J, Nagao K, Tai T et al. Congenital Primary Penile Teratoma in a Child. Urology, 2014;83(6), 1404-06.

8. Saito T. Glomus tumor of the penis. International Journal of Urology, 2000;7(3), 115-17.

9. Grover S, Jha M, Sharma B, Kapoor S et al. Verrucous Hyperplasia: Case report and differential diagnosis. Sultan Qaboos University Medical Journal 2017;17(1), 98-102.

10. Hazarey, V, Ganvir S, Bodhade A. Verrucous hyperplasia: a clinico-pathological study. Journal of Oral and Maxillofacial pathology 2011;15(2), 187-91.

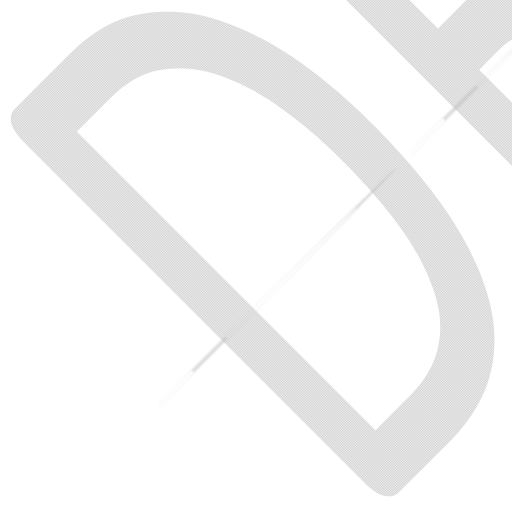




\section{Figures and Tables}

Fig. 1. $1.5 \mathrm{~cm}$ beige ulcerative and crateriform lesion on the right lateral side of the glans
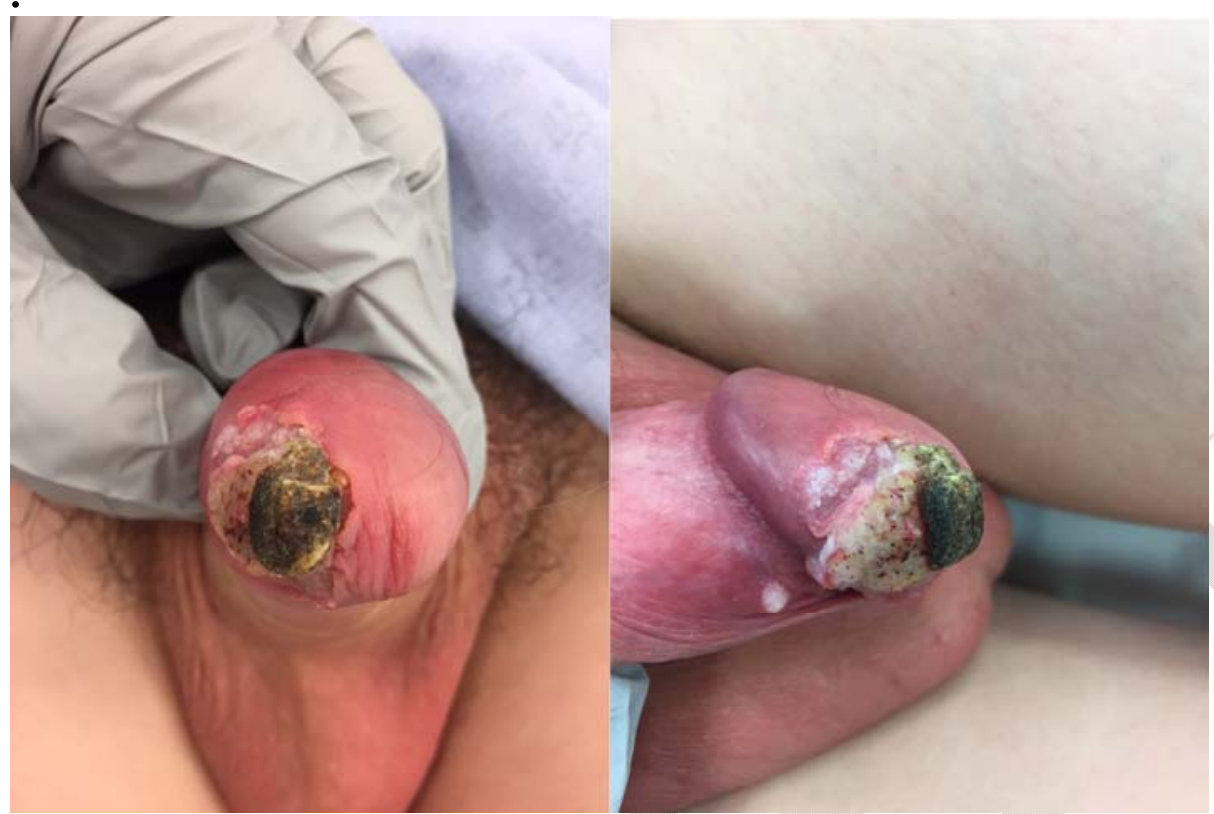

Fig. 2. Histology showed marked epidermal hyperplasia with numerous dyskeratotic cells scattered at different levels of this epidermis. There was no atypia nor mitotic figures or koilocytes.

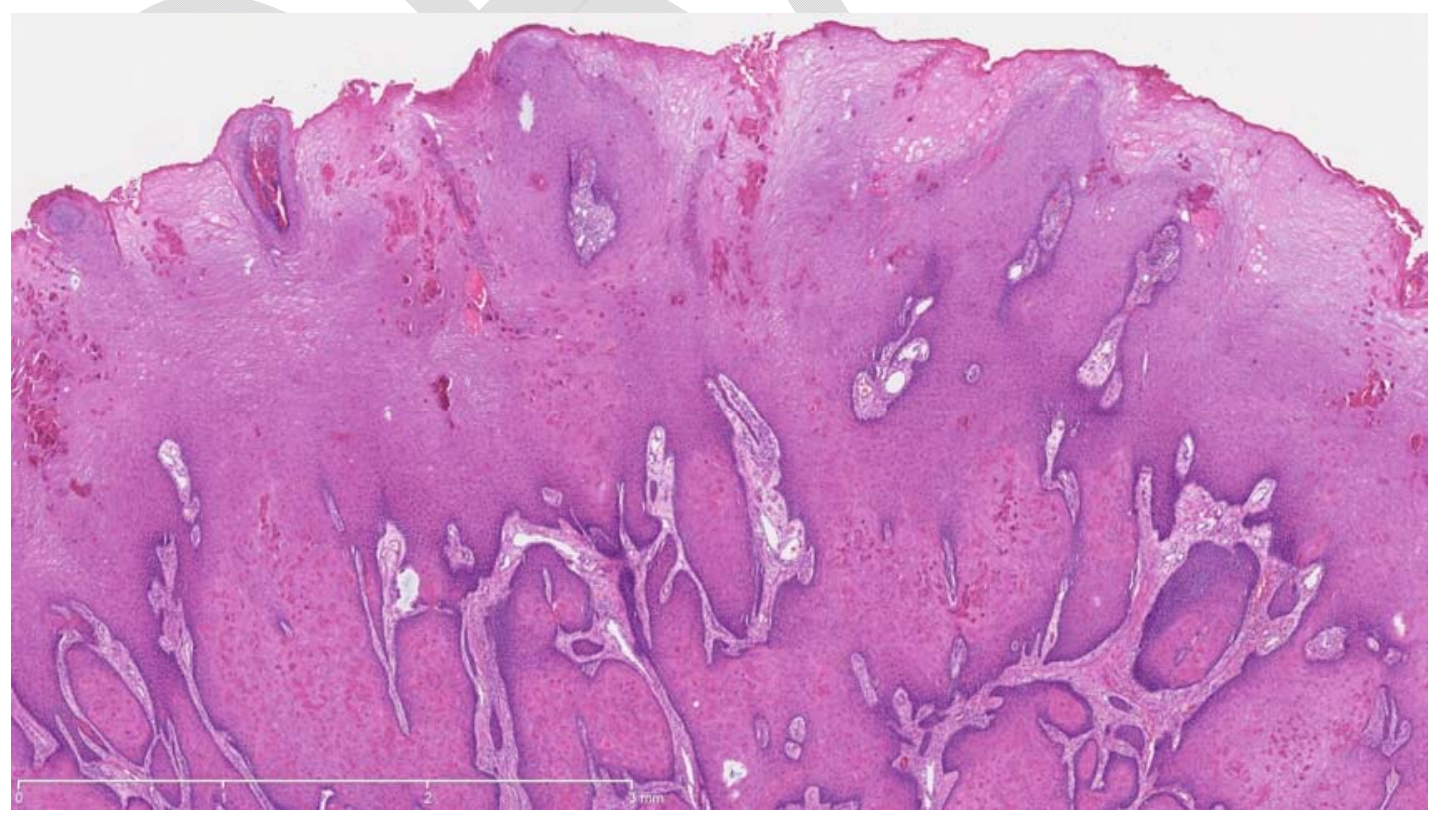


Fig. 3. At one-month followup, two new lesions near the previous surgical excision area were noted.

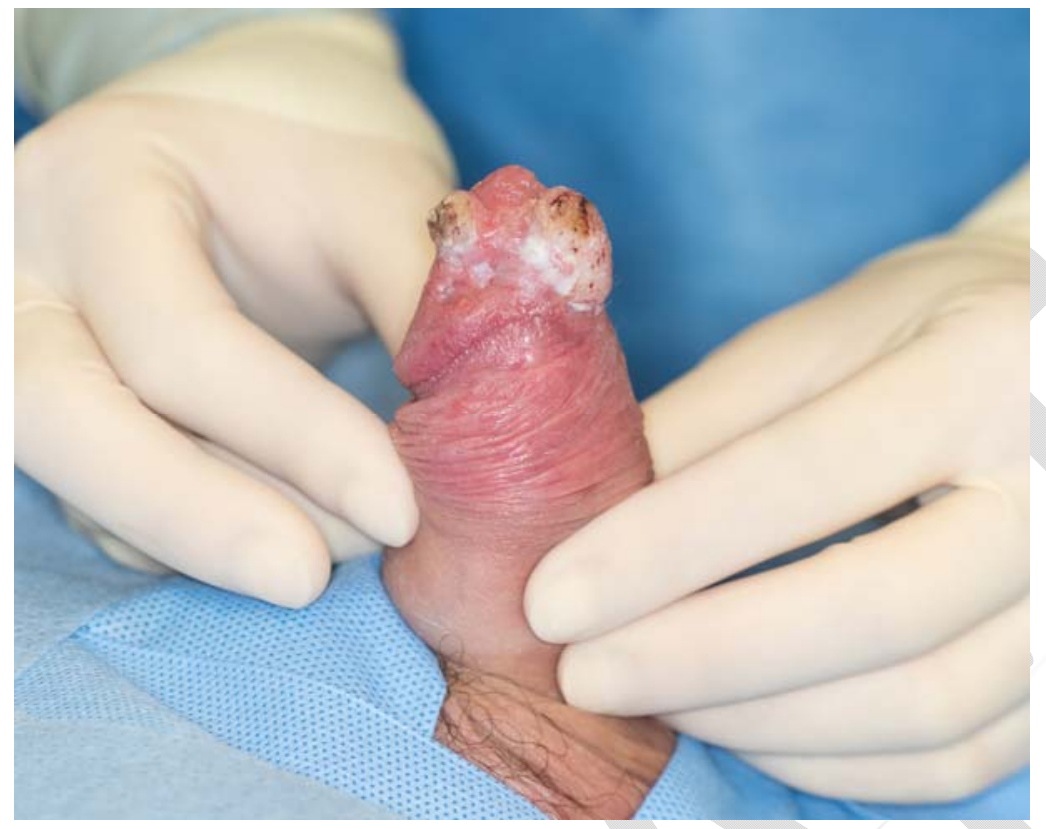

Fig. 4. Two months later, a new lesion appeared on the right side of the glans at great distance from the previous resection sites. Partial penectomy with glans resection and entire corpora cavernosa preservation was performed.

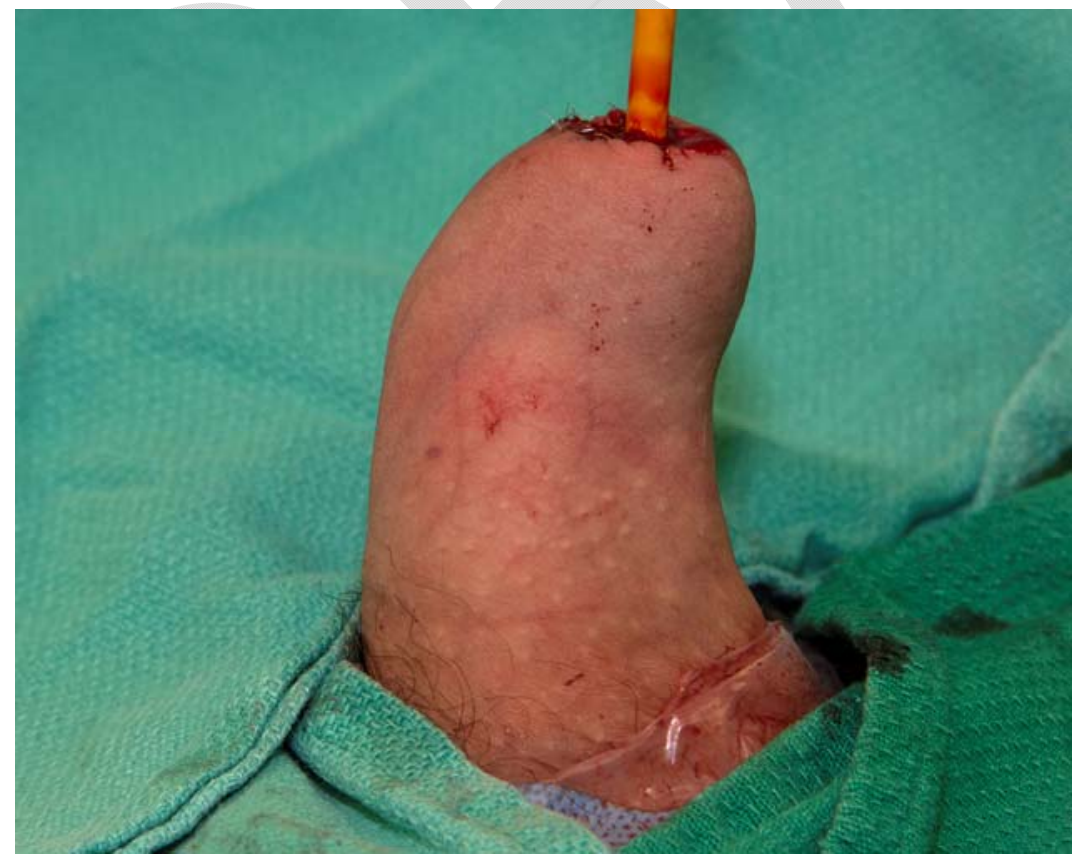

